\title{
Prevalencia de Infección por Virus de Hepatitis B en pacientes pediátricos Hemato-oncológicos.
}

\section{Infection prevalence of Hepatitis B Virus in pediatric hematology-oncology patients}

\section{Karen Anabel Madrid Hernández* Roxana Martínez Beckerat**}

\section{RESUMEN}

Antecedentes y objetivos: El virus de la hepatitis $\mathrm{B}(\mathrm{VHB})$ se transmite por vía sexual y por vía sanguínea. En la mayoría de los brotes conocidos en hospitales el contagio se ha asociado a prácticas poco seguras de inyección, realización de procedimientos, y a la falta de adherencia a las precauciones universales para prevención de infecciones. Por lo cual se quiso determinar la prevalencia de infecciones por VHB, en pacientes Hemato-oncológico sometidos a tratamiento de quimio- terapia y/o transfusión de hemoderivados. Pacientes y Métodos: Este estudio descriptivo detalla la prevalencia y las características clínicas y epidemiológicas de los pacientes de la Sala de Hemato-oncología Pediátrica del Hospital Nacional Dr. Mario Cata rino Rivas (HNMCR), a quienes se les diagnosticó infección VHB, entre 1999-2011. Resultados: La prevalencia de infección por VHB en pacientes hemato-oncológicos fue de un $1.86 \%$, el $64 \%$ de estos pacientes contaban con un esquema de vacunación completo. En todos los pacientes se les diagnosticó primero una patología hemato-oncológica y fue hasta después de un año o más que se realizó el diagnóstico de infección por VHB. Conclusiones: las transfusiones son un factor de riesgo inminente en pacientes que las ameritan sobre todo en los pacientes con diagnósticos hemato-oncológicos.

\section{PALABRAS CLAVE}

Hepatitis B, Infección, Prevalencia.

\section{ABSTRACT}

Background and objectives: The hepatitis $B$

\footnotetext{
* Residente de tercer año de Pediatría, Universidad Nacional Autonoma de Honduras en el Valle de Sula UNAH-VS

** Hemato-oncóloga Pediatra del Hospital Nacional Dr. Mario Catarino Rivas HNMCR

Dirigir correspondencia a: karenmadridh@gmail.com Recibido: 22 de Noviembre 2014, Aprobado: 12 de Marzo 2,015
}

virus (HBV) is transmitted sexually and through blood. In most outbreaks in hospitals, HBV infections have been associated with unsafe injection practices, performing procedures, and lack of adherence to universal precautions for infection prevention. The objective is to determine the prevalence of infections with $\mathrm{HBV}$ in patients undergoing chemotherapy and-/-or blood transfusion. Patients and Methods: This descriptive study details the prevalence, the clinical and epidemiological characteristics of patients in the Pediatric Hematology-oncology ward at the Dr. Mario Catarino Rivas Nacional Hospital (HNMCR) who were diagnosed with HBV infection, between years 1999-2011. Results: The prevalence of HBV infection in hemato-oncological patients was $1.86 \% .64 \%$ of these patients had a complete vaccination. All patients were diagnosed first with hemato-oncological pathology and it was after a year or more that the diagnosis of HBV infection was performed. Conclusions: Transfusions are an imminent risk factor in patients that need it especially in patients with Hematologiconcológic diagnosis.

\section{KEYWORDS}

Hepatitis B, Infection, Prevalence.

\section{INTRODUCCIÓN}

La sobrevida de los pacientes oncológicos ha aumentado con el advenimiento y avance en el tratamiento; sin embargo, la necesidad de terapias intensivas y sustitutivas de la enfermedad misma predispone al paciente a presentar daño orgánico y es quizás el parénquima hepático el más afectado. Unos de los aspectos a considerar es la presencia de infección con hepatitis viral. Los niños que reciben terapia oncológica están expuestos a infectarse o reactivar una infección por virus de hepatitis $B$ (VHB). ${ }^{(1-3)}$ ya que reciben trasfusiones del 
hemoderivados en forma frecuente.

La transmisión horizontal se previene cumpliendo estrictamente las normas de control de infección intrahospitalaria. A pesar de estas medidas, existe un riesgo residual de transmitir esta infección a través de transfusiones de productos sanguíneos, el cual se asocia principalmente al periodo de incubación de la enfermedad. En éste periodo los marcadores serológicos de infección podrian no ser detectados y los donantes de sangre podrán presentar una infección por VHB oculta, caracterizada por ausencia de marcadores serológicos asociadas, por lo general, con una baja carga de ADN. ${ }^{(4-6)}$

La infección por VHB puede dar lugar a una amplia variedad de afecciones que abarcan desde la hepatitis aguda, de duración y gravedad variable, hasta hepatopatía crónica. En pacientes inmunosupresos la probabilidad de desarrollo de infección crónica es mayor. Así también, la probabilidad de cronicidad es inversamente proporcional a la edad: el riesgo de desarrollo de infección crónica de hepatitis B es de hasta un $90 \%$ en los recién nacidos de madres con antígeno de la cubierta de Hepatitis $\mathrm{B}(\mathrm{HBeAg})$ positivo, de 25 a $30 \%$ en niños menores de 5 años y menos de $5 \%$ en adultos. La infección por VHB en individuos con cáncer se caracteriza por una alta tasa de cronicidad y se relaciona con mayor riesgo de daño hepático, de desarrollar un cáncer secundario, mayor posibilidad de recaída de su enfermedad oncológica, un riesgo epidemiológico especial para sus familiares y los trabajadores de la salud que atienden a estos pacientes $y$ además deterioro en la calidad de vida de los sobrevivientes. ${ }^{(7-9)}$

Durante los últimos años se ha evidenciado la presencia de infección por virus de hepatitis $B$ en niños hospitalizados en el Servicio de Hemato-oncología del Departamento de Pediatría del Hospital Nacional Dr. Mario Catarino Rivas. Utilizando métodos serológicos para detectar los antígenos de superficie y especifico ( $\mathrm{HBsAg}$ y HBeAg). Así como la detección de anticuerpos contra el antígeno de superficie (anti-HBs), anticuerpo IgM contra HBc y anticuerpo contra el antígeno e.(10-12) Por consiguiente, a través de este estudio se pretende determinar la prevalencia de infecciones por VHB, en pacientes hemato-oncológicos sometidos a tratamiento de quimioterapia y/o transfusión de hemoderivados.

\section{PACIENTES Y MÉTODOS}

Luego de la aprobación del comité de ética se realizó un estudio descriptivo donde se detalla la caracterización clínica y epidemiológica de los pacientes de la Sala de Hemato-oncología Pediátrica del Hospital Nacional Dr. Mario Catarino Rivas a quienes se les diagnosticó infección por VHB.

Se obtuvo el consentimiento informado de los padres o representantes legales previo a obtener la información de los expedientes clínicos tanto de las áreas de hospitalización como de la consulta externa. Se incluyeron en el análisis únicamente a aquellos pacientes que tuvieron como diagnóstico cualquier patología oncológica más infección VHB confirmado por serología (cargas virales y HBsAg). A partir de 1999 hasta el 2011. Se encontró registro de 1,341 pacientes en el servicio de Hemato-oncología pediátrica, de los cuales 25 cumplen con los criterios de inclusión. Se excluyeron los casos con infección por VIH.

Los datos obtenidos se analizaron y procesaron en el software estadístico EPI-INFO versión 7. Realizando el análisis de variable con medidas de frecuencia (porcentajes y tasas). Así como también se utilizó la hoja de cálculo Microsoft Office Excel XP-2007, para organizar y presentar los datos.

\section{RESULTADOS}

En las características epidemiológicas de género y edad de los 25 pacientes el $40 \%$ se encontraban entre los 13-16 años de edad, sin tener una variación significativa de género. (Ver tabla No. 1). 
Tabla No. 1: Características epidemiológicas de pacientes hemato-oncológicos e infección por $\mathrm{VHB}$

\begin{tabular}{lcc}
\hline $\begin{array}{l}\text { Características } \\
\text { Epidemiológicas }\end{array}$ & $\mathbf{n = 2 5}$ & porcentaje \\
\hline Edad (años) & & \\
$\leq 4$ & 0 & 0 \\
$5-8$ & 6 & 24 \\
$9-12$ & 4 & 16 \\
$13-16$ & 10 & 40 \\
$17-20$ & 3 & 12 \\
$\geq 21$ & 2 & 8 \\
Género & 13 & 52 \\
$\quad$ Femenino & 12 & 48 \\
Masculino & 12 & \\
\hline
\end{tabular}

VHB Virus Hepatitis B

Fuente: instrumento "Infección VHB-paciente Hemato-oncológico"

En el $28 \%$ de los casos se realizó el diagnóstico infeccioso después de haberse efectuado el diagnóstico hemato-oncológico como se muestra en la tabla No. 2.

Se encontró que un $64 \%$ de los pacientes contaban con un esquema de vacunación completo, $16 \%$ incompleto y $20 \%$ desconocía su esquema, coincidiendo este último porcentaje con los pacientes de mayor edad.

La patologías hemato-oncológicas en la que se encontró infección con VHB fue Leucemia linfocítica aguda (ver gráfico No. 1). Además de ser la patología que más procedimientos invasivos tuvo y en quienes más se transfundió hemoderivados.

Tabla No. 2: Tiempo transcurrido entre el diagnóstico hemato-oncológico e infección por VHB

\begin{tabular}{lcc}
\hline $\begin{array}{l}\text { Tiempo trans- } \\
\text { currido (años) }\end{array}$ & $\mathbf{n = 2 5}$ & porcentaje \\
\hline 1 & 3 & $12 \%$ \\
2 & 7 & $28 \%$ \\
3 & 6 & $24 \%$ \\
4 & 3 & $12 \%$ \\
NC $^{*}$ & 6 & $24 \%$ \\
\hline
\end{tabular}

Hemato-oncológico" *NC= no consignado

Fuente: instrumento "Infección VHB-paciente
Gráfico No. 1: Diagnóstico hemato-oncológico e infección VHB

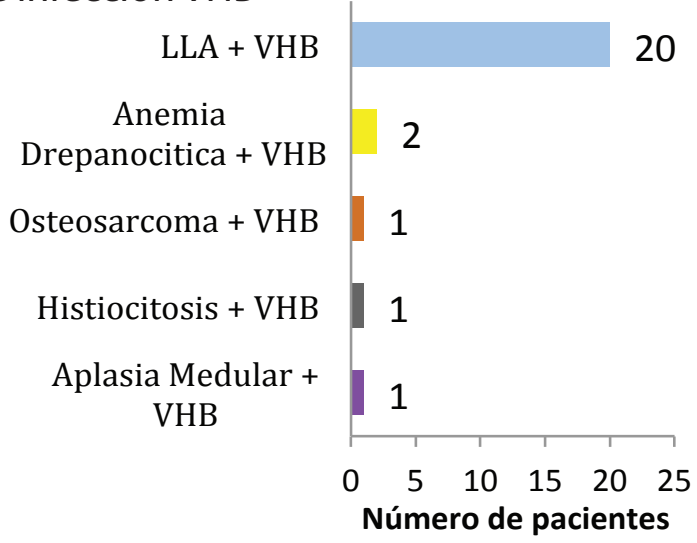

LLA: Leucemia linfocítica aguda, VHB: Virus de Hepatitis B Fuente: instrumento "Infección VHB-paciente Hematooncológico"

Se les realizó biopsia hepática al $8 \%(n=2)$ de los casos. El tratamiento recibido en la totalidad de los pacientes fue Lamivudina como monoterapia. El 52\%, de estos pacientes desarrollaron resistencia ocupando doble terapia (Lamivudina/Tenofovir o Interferón/Lamivudina) y el 4\% llegó a necesitar triple terapia (Lamivudina, Tenofovir, Interferón).

\section{DISCUSIÓN}

Los niños en tratamiento antineoplásico se consideran una población de mayor riesgo a contraer la infección por VHB debido a las frecuentes transfusiones de hemoderivados $y$ la realización de procedimientos para recibir tratamiento o para toma de muestras y que están asociados con la inoculación parenteral del virus como son la biopsia de médula ósea, venoclisis y punciones lumbares. ${ }^{(9,12)}$ El $100 \%$ de los pacientes estudiados recibieron hemoderivados en algún momento del transcurso de su enfermedad como también se les realizó procedimientos invasivos en más de una ocasión previo al diagnóstico Hepatitis $B$.

Dada la inmunosupresión a la cual se encuentran expuestos estos pacientes oncológicos, se ha demostrado una disminución marcada en protección brindada por las vacunas incluyendo la de la Hepatitis $B$, haciéndolos más vulnerables ante la exposición del virus. ${ }^{(12,13)}$ 
El 64\% tenía el esquema de vacunación contra el VHB completo. Sin embargo $20 \%$ desconocían datos de vacunación sobre todo los pacientes mayores de 4 años. Ninguno de los padres de los pacientes tenía esta infección.

La prevalencia de la infección por VHB en pacientes con diagnóstico hemato-oncológico es de $1.86 \%$, similar a estudios reportados en Latinoamérica. ${ }^{(14)}$ La leucemia linfocítica aguda fué el diagnóstico oncológico con mayor prevalencia, probablemente asociado a las múltiples transfusiones de hemoderivados recibidas.

La infección por VHB en individuos con cáncer se caracteriza por una alta tasa de cronicidad y se relaciona con mayor riesgo de daño hepático. ${ }^{(15,16)}$ La biopsia hepática es el método diagnóstico preciso en estos casos. ${ }^{(17,18)}$ Solo al $8 \%$ (2 pacientes) se les pudo realizar biopsia hepática por falta de recursos.
En la actualidad el Interferón y la Lamivudina son las únicas drogas aprobadas por la administración de alimentos y drogas de Estados Unidos (FDA) para niños con Hepatitis B. ${ }^{(19-21)}$ La totalidad de los pacientes recibieron tratamiento con monoterapia (Lamivudina). Hubo alta resistencia al tratamiento ya que el $52 \%$ ocuparon doble terapia (Lamivudina/Tenofovir o Interferón/Lamivudina) y triple terapia (Lamivudina, Tenofovir, Interferón).

A pesar del intento por mantener un control de calidad efectivo en los hemoderivados, las transfusiones siguen siendo un factor de riesgo inminente en pacientes que las necesiten sobre todo en los pacientes con diagnósticos hemato-oncológicos. Es necesario mejorar la vigilancia de complicaciones por transfusiones con la medición de niveles de anticuerpos y antígenos para VHB y biopsia hepática, así poder definir la necesidad de refuerzos de la vacuna contra VHB. Y hacer un estudio dirigido a definir la terapia apropiada para nuestros pacientes debido al alto grado de resistencia al tratamiento.

\section{BIBLIOGRAFÍA}

1. Jessi Erlichman, Abdul Salam, Bárbara A. Haber. Use of Complementary and Alternative Medicine in Pediatric Chronic Viral Hepatitis. Hepatology and Nutrition JPGN 2010; 50:417-421.

2. Brook G, Soriano V, Bergin C. European guideline for the management of hepatitis $B$ and $C$ virus infections, 2010 International Journal of STD \& AIDS 2010; 21:669-678.

3. Chronic Hepatitis Working Group: Murray KF, Shah U, Moham N, Heller S, Gonzales Peralta RP, Kelly D, et al. Chronic Hepatitis. Journal of Pediatric and Gastroenterology and Nutrition. 2008. 47: 225-233.

4. Maureen M Jonas. Children's Hospital, Harvard Medical School, Boston, MA. Treatment of Chronic Hepatitis B in Children.
Journal of Pediatric Gastroenterology and Nutrition 2006.43:56-60.

5. Uzma Shah, Deirdre Kelly, Mei-Hwei Chang, Tomoo Fujisawa European Society for Pediatric Gastroenterology, Hepatology, and Nutrition and North American Society for Pediatric Gastroenterology, Hepatology, and Nutrition. Management of Chronic Hepatitis B in Children. Journal of Pediatric Gastroenterology and Nutrition 2009.48: 399-404.

6. Byung Ho Choe, Ji Hye Lee, You Cheol Jang, Chang Hwan Jang, Ki Won Oh, Soonhak Kwon, et al.: Long-term Therapeutic Efficacy of Lamivudine Compared With Interferon-a in Children with Chronic Hepatitis B: The Younger the Better. Journal of Pediatric Gastroenterology and Nutrition 2007. 44: 92-98. 
7. Laubscher B, Gehri M, Roulet M, Wirth S, Gerner P. Survival of Infantile Fulminant Hepatitis B and Treatment with Lamivudine. Journal of Pediatric Gastroenterology and Nutrition April 2005.40:518-520.

8. Neelam Mohan, González-Peralta RP, Tomoo Fujisawa, Mei-Hwei Chang, Solange Heller, Davison SM, et al. Chronic Hepatitis C Virus Infection in Children JPGN February 2010; 50 (2):123-131.

9. Marcellin P, Chang T-T, Lim SG, Tong MJ, Sievert W, Shiffman M. et al. Adefovir Dipivoxil for the Treatment of Hepatitis B e Antigen-Positive Chronic Hepatitis B. N Engl J Med 2003; 348:808-16.

10. Maurin Espinosa-Holguin, Melvis Arteaga-Vizcaina, Leticia Puerto. Hepatitis B en niños con Cáncer. Revista Gastroenterologia de Peru 2006; 26(3):23-34.

11. Kose S, Olmezoglu A, Gozaydin A, Ece G. Seroprevalence of Hepatitis B and C among Oncology Patients in Turkey. J Health Popul Nutr. [Citado 2014, diciembre 13] 2011; 29(6): 652-655. Disponible en: http:// www.ncbi.nlm.nih.gov/pmc/articles/ PMC3259729/.

12. Buster eh, Vander Eijk aa, de Man Ra, Schalm sw. Doctor-to- patient transmission of hepatitis B virus: The Potential of Antiviral Therapy for Prevention. Scand J Gastroenterol. 2004; 241: (Suppl) 45-9.

13. Custer B, Sullivan SD, Hazlet TK, Iloeje U, Veenstra DL, Kowdley KV. Global epidemiology of hepatitis B virus. J Clin Gastroenterol 2004; 38(Suppl 10):158-68.

14. Zubieta M, Santolaya ME, Hurtado C, Alvarez AM, Aviléz AM, Becker A et. al.: Seroprevalencia de virus hepatitis $B$ en niños con cáncer en tratamiento quimioterápico en 6 hospitales de Santiago de Chile. Rev. méd. Chile [online].2009, vol.137, n.7, pp. 906-911. ISSN 0034-9887. http://dx.doi.org/10.4067/S0034-98872009 000700007.

15. Márquez Lucia, Salas Albermary, Moschella Filomena, Artís, María Teresa. Seroprotección contra el virus de hepatitis $b$ en Pacientes pediátricos con cáncer. 2010; 64(4): 27-44.

16. Larry K. Pickering, Carol J. Backer, Sarah S. Long, Julia A. Mc Millan. Red Book Enfermedades Infecciosas en Pediatría. 27 Edición. Argentina: médica Panamericana, 2008. P 430-439.

17. Ke- Qin Hu, Calvin Pan. Advances in Diagnosing and Managing Hepatitis B Virus (HBV) Infection. North American Journals of Medicine and Science. 2011;4

18. Kalyan Ram Bhamidimarri, Calvin Pam. Hepatitis B Reactivation during Immunosuppression: From Pathogenesis to Management Strategy. Advances in Diagnosing and Managing Hepatitis B Virus (HBV) Infection N A J Med Sci. 2011; 4(1):44-49.

19. Nermin N Salkica, Muharem Zildzica, Kasim Muminhodzica,Nada Pavlovic-Calica, Enver Zerema, Sead Ahmetagicb, et al.: Intrafamilial transmission of hepatitis B in Tuzla region of Bosnia and Herzegovina. European Journal of Gastroenterology \& Hepatology 2007, 19: 113-118

20. Buster EH, Van Der Eijk AA, De Man RA, Schalm SW.: Doctor-to-patient transmission of hepatitis $B$ virus: the potential of antiviral therapy for prevention. Scand J Gastroenterol (Suppl) 2004; 241:45-9.

21. Craxi A, Tine F, Vinci M Almasio P, Camma C, Garofalo ,Pagliaro L.: Transmission of hepatitis delta viruses in the households of chronic hepatitis $B$ surface antigen carriers: a regression analysis of indicators of risk. Am J Epidemiol 1991; 134:641- 650. 